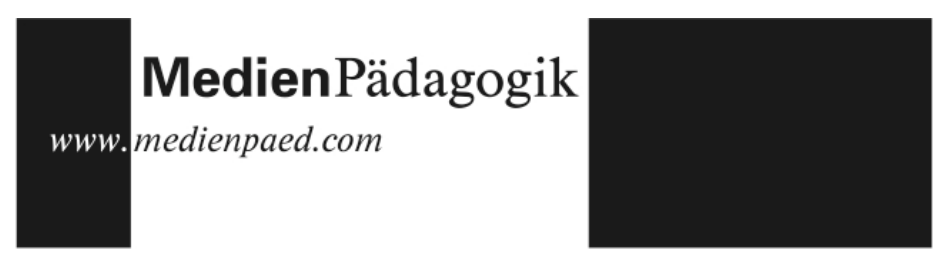

Rezensionen

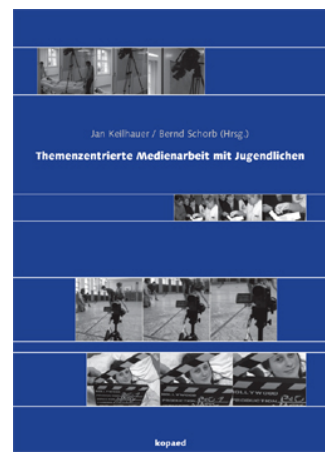

Jan Keilhauer und Bernd Schorb

Themenzentrierte Medienarbeit mit Jugendlichen

Ein Modellprojekt mit deutschen und tschechischen Jugendlichen

zum Thema Präimplantationsdiagnostik

München: kopaed, 2010. 115 Seiten

ISBN: 978-3-86736-241-2

$€ 14,80$; CHF $18,95-$

\title{
Ein pädagogischer Synergieeffekt für Praxis und Theorie: Themenzentrierte Medienarbeit
}

Aktive Medienarbeit als bildungspraktische Methode und «Königsweg» der (handlungsorientierten) Medienpädagogik (Schell 1993) ist nicht nur in der medienpädagogischen Theorie sondern auch in der pädagogischen Arbeit mittlerweile fest etabliert. Allein ihre Fokussierung auf die Förderung von Medienkompetenz als unabdingbare Handlungskompetenz in mediatisierten Lebenswelten unterstreicht ihre - nicht nur pädagogische - Bedeutung.

Jan Keilhauer und Bernd Schorb stellen nun anhand des mit deutschen und tschechischen Schüler/innen durchgeführten Modellprojektes PID - Perspektiven im Diskurs eine Erweiterung der aktiven Medienarbeit (Schell 1993) bzw. reflexiv-praktischen Medienarbeit (Schorb 1995) als themenzentrierter Medienarbeit vor. Im Mittelpunkt steht dabei nicht mehr primär die Förderung von Kompetenzen im Umgang mit Medien, sondern vielmehr «die Bearbeitung gesellschaftlicher Themenbereiche" (Themenzentrierte Medienarbeit mit Jugendlichen 10) mittels Medien. In drei Teilen wird diese neue medienpädagogische Methode für die Bildungspraxis in ihren theoretischen Fundierungen wie auch ihrer praktischen Anwendung dargelegt.

Als Ausgangsposition erläutern Keilhauer und Schorb im ersten Teil des Bandes, warum gerade Medien geeignet sind, Jugendliche zu einer Auseinandersetzung mit Themen von gesellschaftlicher Relevanz anzuregen. In enger Verschränkung mit der alltäglichen Medienpraxis der Jugendlichen wird an vertraute Handlungsweisen angeknüpft und selbstbestimmtem Lernen durch die stets geforderten individuellen Entscheidungen im Prozess 


\section{Rezensionen}

der Medienproduktion ermöglicht. Auf diese Weise - und das ist die Grundidee des Ansatzes - werden Jugendlichen Räume zur Partizipation an gesellschaftlichen Diskursen eröffnet. Ungeachtet der Fokussierung auf eine themeninitiierte Medienarbeit gerät Medienkompetenz als Zieldimension dabei nicht ins Hintertreffen, sondern ist «eng mit den themenbezogenen Zielen verbunden» (Themenzentrierte Medienarbeit mit Jugendlichen 10).

Der im zweiten Teil des Bandes explizierte theoretische Bezugsrahmen der themenzentrierten Medienarbeit rekapituliert jenen der reflexiv-praktischen Medienarbeit und erstreckt sich damit vielfältig vom Medienkompetenzbegriff (Schorb 2005) über kommunikative Kompetenz (Baacke 1973) hin zu handelndem Lernen (Dewey 1993). Auf dieser Grundlage wird die themenzentrierte Medienarbeit als (medien-)pädagogische Methode entfaltet. Sowohl Ziele und Kompetenzdimensionen als auch Prinzipien und Phasen der Projektarbeit finden hier eine nachvollziehbare Darstellung. Indem diese Ausführungen einen systematischen und strukturierten Aufbau des (medien)pädagogischen Lehr- und Lernprozesses ermöglichen, bieten sie gleichsam eine Anleitung für bildungspraktisches Handeln. Besonders deutlich wird dabei, wie Professionalität im medienpädagogischen Handeln durch theoretisch fundierte und damit dezidierte Lernziele erreicht werden kann.

Ausgehend von der so referierten theoretischen Basis, die medienpädagogischen Projekten häufig fehlt (Kübler 2002), vertiefen verschiedene am Projekt PID - Perspektiven im Diskurs beteiligte Akteure im dritten Teil des Buches die bildungspraktische Perspektive der Methode. Die konkreten Inhalte des Projektes werden dabei so abstrahiert, dass «sie als Leitfaden für eigene Projekte herangezogen werden können» (Themenzentrierte Medienarbeit mit Jugendlichen 23). Die Ausführungen zu Ablauf und Spezifika des Projektes sind systematisch ergänzt um Reflexionen, die Möglichkeiten und Grenzen der themenzentrierten Medienarbeit innerhalb und außerhalb des schulischen Raumes aufzeigen. Nicht zuletzt in der transnationalen Ausrichtung des Projektes ist eine seiner Stärken begründet: die Vielfalt der pädagogischen Einsatzmöglichkeiten des Ansatzes. 
Der Band schließt mit einer sehr präzisen Darstellung der projektspezifisch erarbeiteten Medien-Kompetenzen, die noch einmal unterstreicht, dass Medienkompetenzförderung ein wichtiger Bestandteil der themenzentrierten Medienarbeit ist und damit die Relevanz dieser Methode für die Bildungspraxis der Medienpädagogik bestätigt. Wünschenswert wäre an dieser Stelle indes ein direkter Bezug zu den Kompetenzdimensionen, wie sie in den theoretischen Grundlegungen zur themenzentrierten Medienarbeit entfaltet wurden.

Themenzentrierte Medienarbeit als medienpädagogische Projektmethode und theoretisches Konzept besticht durch die Synergie von Praxis und Theorie. Damit konsolidiert sie die Bedeutung einer theoretischen Fundierung der medienpädagogischen Praxis. Themenzentrierte Medienarbeit zeigt die Möglichkeiten für eine Konkretisierung von medienpädagogischem Handeln und ist (hier als Lektüre) gleichermaßen für Wissenschaft und Bildungspraxis interessant.

Theresa Schmidt

\section{Literatur}

Baacke, Dieter. Kommunikation und Kompetenz. Grundlegung einer Didaktik der Kommunikation und ihrer Medien. München: Juventa, 1973.

Dewey, John. Demokratie und Erziehung. Eine Einführung in die philosophische Pädagogik. Weinheim: Beltz, 1993.

Kübler, Hans-Dieter. Medienpädagogik in der Informationsgesellschaft. In: Paus-Hasebrink, Ingrid; Lampert, Claudia; Süß, Daniel. Medienpädagogik in der Kommunikationswissenschaft. Wiesbaden: Westdeutscher Verlag, 2002, S.169-190.

Schell, Fred. Aktive Medienarbeit. Theorie und Praxis. München: kopaed, 1993.

Schorb, Bernd. Medienalltag und Handeln. Medienpädagogik in Geschichte, Forschung und Praxis. Opladen: Leske + Budrich, 1995.

Schorb, Bernd. Medienkompetenz. In: Hüther, Jürgen; Schorb, Bernd. Grundbegriffe Medienpädagogik. München: kopaed. 2005, S. 257-262. 